\title{
Kaempferol conjugated gold nanoclusters enabled efficient for anticancer therapeutics to A549 lung cancer cells
}

This article was published in the following Dove Press journal: International Journal of Nanomedicine

\author{
Saravanan Govindaraju (D) ${ }^{1, *}$ \\ Arivazhagan Roshini ${ }^{2} *$ \\ Min-Ho Lee ${ }^{3}{ }^{3}$ \\ Kyusik Yun $\mathbb{D}^{\prime}$
}

'Department of Bionanotechnology, Gachon University, Gyeonggi-do 13120, Republic of Korea; ${ }^{2}$ Department of Neuroscience and Physiology, SUNY Upstate Medical University, Syracuse, NY 13210, USA; ${ }^{3}$ School of Integrative Engineering, Chung-Ang University, Seoul 06974, Republic of Korea

*These authors contributed equally to this work
Correspondence: Min-Ho Lee School of Integrative Engineering, ChungAng University, Seoul 06974, Republic of Korea

Emailmhlee7@cau.ac.kr

Kyusik Yun

Department of Bionanotechnology, Gachon University, Gyeonggi-do 13120, Republic of Korea

Email ykyusik@gachon.ac.kr
Background: Kaempferol $(\mathrm{K})$ is a recognized anticancer drug that can conjugate with small-size gold nanoclusters (AuNCs).

Materials and methods: K-AuNCs were synthesized and their use as an anticancer drug was explored using A549 lung cancer cells. Colony formation and cell migration assays were carried out. The morphology of the K-AuNCs treated A549 cells was explored using bioatomic force microscopy.

Results: The K-AuNCs were 1-3 nm in diameter and emitted strong fluorescent at $650 \mathrm{~nm}$ following excitation at $550 \mathrm{~nm}$. The stretching and bending nature of the K-AuNCs were analyzed by the Fourier transform infrared spectroscopy. The presence of kaempferol in the AuNCs were confirmed by the PL spectroscopy.

Conclusion: The synthesized K-AuNCs mainly targeted and damaged the nuclei of the cancer cells. This composite nanocluster was less toxicity to the normal human cell and higher toxicity to the A549 lunch cancer cell and these material is potential for anticancer drug delivery and bio imaging applications.

Keywords: gold cluster, fluorescent property, biocompatible, flavonoids, anticancer activity

\section{Introduction}

Cancer is an abnormal, uncontrolled division and growth of cells or tissues that causes major health problems and leads to death. Tumor are highly complicated error and has high mortality rate. ${ }^{1}$ The World Health Organization estimates that approximately 84 million people die each year from cancer. ${ }^{2}$ Current drugs and therapy have a number of complications including lack of drug effectiveness, selectivity, and side effects. More effective drugs are needed for cancer treatment. Over the past few decades, cancer treatments have developed using natural products and their derivatives. ${ }^{3}$ Most recently, nanomaterials gain more attention with the development of multifunctional nanomaterials processing both anticancer property and bioimaging with less toxicity to normal cells. ${ }^{4-7}$

Flavonoids are polyphenolic compounds that are abundant in tea, certain vegetables and fruits including broccoli, apples, beans, and strawberries. ${ }^{8}$ The flavonoids have antioxidant and anti-inflammatory properties and it is potentially cytotoxic to the cancer cells. Among the recently found polyphenols, kaempferol has demonstrated to regulate several types of tumor progression. Many studies have reported the potential anticancer therapy of kaempferol, and it has proven to cause cell cycle arrest in various cancer types including lung, breast, pancreatic, ovarian, chronic 


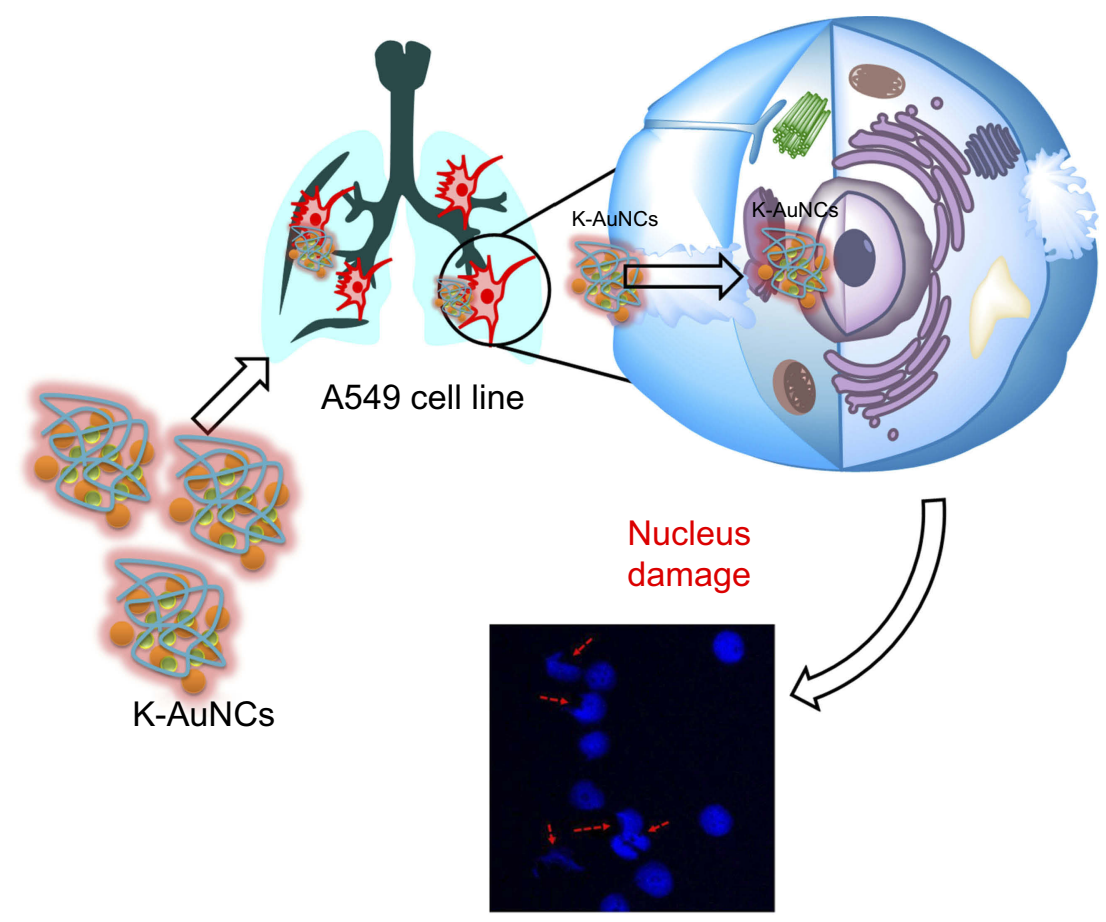

Scheme I Schematic diagram of K-AuNCs for anticancer therapy.

myelogenous leukemia, and colon cancer. ${ }^{9,10}$ Kaempferol elicits anticancer activity by inhibiting phosphatidylinositol-3-kinase (PI3K) and ribosomal s6 kinase. Kaempferol has an integrated $\pi$ bond and strongly binds with oxygen atoms, and features a steric configuration that easily interacts with metal ions. ${ }^{11}$

Several different types of nanomaterials are being used for drug delivery such as gold (Au), silver, copper, and magnetic nanoparticles (NPs). These NPs are biocompatible, easily degradable, and less toxic to normal cells. Among these, gold nanomaterials gain more attention in cancer drug delivery, bioimaging and gene delivery due to the low cytotoxicity, easy synthesis procedures, and can be easily formulated with other drugs. ${ }^{12}$ A noble metal nanocluster (NC) for example, AuNCs consists of 1 to 10 number of atoms combined to form clusters. These NCs are ultra-small compared to the Fermi wavelength of free electrons. ${ }^{13}$ AuNCs have unique physicochemical properties, are easy to synthesize, and are stable. These attributes have been exploited in diagnosis and imaging. ${ }^{14}$ Compared to NPs, $\mathrm{NCs}$ are less toxic due to their biocompatible synthesis methods, uniform size, and surfactant property. Natural drugs like flavonoids conjugated with NCs can be applied in anticancer therapy and bioimaging. ${ }^{15}$ AuNCs are approximately $2 \mathrm{~nm}$ in size. The strong fluorescent property of them makes them attractive for diagnosis and imaging, permits targeting cell nuclei with single-particle sensitivity.
In this study, we synthesized kaempferol-conjugated AuNCs (K-AuNCs) using bovine serum albumin (BSA) as a stabilizing agent. To our knowledge, this is the first report on K-AuNCs. The synthesized K-AuNCs were characterized with UV-vis spectroscopy, PL spectroscopy, FTIR, TEM, and Bio-AFM. This new approach will help carry the development of cancer drug delivery and bioimaging to the next level. As synthesized material is biocompatible to normal cells and cytotoxic only against the lung cancer cells, the synthesized K-AuNCs displayed strong red fluorescence, were active against the A549 lung cancer cells controlling the major hallmarks of cancer, namely, cell proliferation and migratory properties (Scheme 1).

\section{Materials and methods}

Gold(III) chloride trihydrate $\left(\mathrm{HAuCl}_{4} \cdot 3 \mathrm{H}_{2} \mathrm{O}\right)$ and sodium hydroxide $(\mathrm{NaOH})$ were purchased from Sigma-Aldrich (USA). Kaempferol was purchased from Nanjing spring and autumn biological engineering (China). The molecular weight of kaempferol is C15H1006, CAS No-520183, molecular weight is 286.23 , and purity is HPLC $98 \%$. Bovine serum albumin (BSA) was purchased from Millipore LEE (South Korea).

\section{Instrumentation}

Ultraviolet-visible (UV-vis) spectra were obtained using a Cary 100 UV-Vis spectrophotometer (Varian, USA). 
$\mathrm{K}$-AuNCs were highly diluted in distilled water and dark-field images were collected by a UV illuminator (Photon Technology International, USA) at $365 \mathrm{~nm}$. Fluorescence spectra of K-AuNCs were recorded using a QuantaMaster fluorescence spectrometer (Photon Technology International) equipped with an A-1010B Arc Lamp Housing xenon lamp (Photon Technology International), monochromator, and power supply (Brytexbox) with an excitation wavelength of $450 \mathrm{~nm}$. High-resolution transmission electron microscopy (HRTEM) images were taken using a Tecnai ${ }^{\mathrm{TM}}$ G2 F30 Series microscope. Ultrathin carbon type-A, 400 mesh copper grids were used for sample preparation for HRTEM. Fourier-transform infrared spectroscopy (FTIR) was performed using a vortex high resolution 70 FTIR spectrometer (Bruker, USA) equipped with a micro plate extension HTS-XT and attenuated total reflectance units (Bruker). Life time decay was recorded using an EL series of nanosecond pulsed LEDs (Photon Technology International) designed for the EasyLife II with auto-adjustable excitation wavelengths ranging from 260 to $650 \mathrm{~nm}$. A549 cell morphology was measured using a NanoWizard BioScience atomic force microscope (JPK Instruments, USA).

\section{Synthesis of K-AuNCs}

A stock solution of $10 \mathrm{mg}$ kaempferol was dissolved in 5 $\mathrm{mL}$ of $70 \%$ ethanol. In a typical experiment, the prepared kaempferol solution and $5 \mathrm{~mL}$ of $10 \mathrm{mM} \mathrm{HAuCL}_{4}$ were mixed together and heated at $40^{\circ} \mathrm{C}$ with stirring at 800 $\mathrm{rpm}$. Bovine serum albumin $(5 \mathrm{~mL}, 50 \mathrm{mg} / \mathrm{mL})$ was added to the solution. The reaction was maintained for 15 mins, followed by the addition of $500 \mu \mathrm{L}$ of $\mathrm{NaOH}$ solution. The solution was maintained at $50^{\circ} \mathrm{C}$ in an oven with vigorous and continuous stirring for $1 \mathrm{hr}$. During the reaction, the light-yellow color changed to brown, which confirmed the formation of K-AuNCs. The powder samples were collected using a freeze-drier.

\section{In vitro cytotoxicity assay}

The cytotoxicity of the formulation was accessed in HK-2 normal human kidney cells and A549 human lung cancer cells. All the cell lines used in this study were purchased from the American type culture collection (USA). Cells were grown in RPMI-1640 medium supplemented with $10 \%$ fetal bovine serum (FBS) and 1\% penicillin/streptomycin at $37^{\circ} \mathrm{C}$ in an atmosphere of $5 \% \mathrm{CO}_{2}$ in a humidified incubator. To prepare cells for the experiments, the cells were washed, trypsinized, and seeded in 96-well plate at a density of $1 \times 10^{4}$ cells per well in RPMI-1640 media containing $10 \%$ FBS and 1\% penicillin/streptomycin and incubated overnight. The treatment involved two different concentrations of the K-AuNC formulation (12.5 and 25 $\mu \mathrm{g} / \mathrm{mL}$ ) in RPMI-1640 medium. The HK-2 and A549 cells were suspended in media containing specified concentrations of the NCs and incubated for $24 \mathrm{hrs}$. Later, $20 \mu \mathrm{L}$ of the 3-(4,5-dimethylthiazol-2-yl)-2,5-diphenyl tetrazolium bromide (MTT) reagent was added to each well. Media was removed after $4 \mathrm{hrs}$ incubation at $37^{\circ} \mathrm{C}$. Following this, $100 \mu \mathrm{L}$ of dimethylsulfoxide (DMSO) was added to each well, kept of a shaker to dissolve the MTT reagent. The optical density (OD) was measured at $570 \mathrm{~nm}$ using a micro-plate reader.

\section{Nuclear damage}

A549 cells were seeded on a coverslip in a $35-\mathrm{mm}$ dish and allowed to adhere for $24 \mathrm{hrs}$. The cells were then treated with specified concentration of K-AuNCs for $24 \mathrm{hrs}$ at $37^{\circ} \mathrm{C}$. The cells were then fixed with $100 \%$ methanol for 20 mins, washed three times with $1 \times$ Dulbecco's phosphatebuffered saline (DPBS), and stained with DAPI for $20 \mathrm{~s}$. The cells were washed again three times with $1 \times$ DPBS and mounted on a coverslip. The nuclear morphology was examined using confocal laser scanning microscopy.

\section{Colony formation assay}

A549 cells were harvested during the log phase of growth and 500 cells were plated in a $60-\mathrm{mm}$ dish. After $24 \mathrm{hrs}$, fresh media was replaced with or without desired concentration of K-AuNCs and incubated. After two weeks, the cells were washed in $1 \times$ DPBS and fixed with $100 \%$ methanol for 15 mins. The fixed cells were further washed twice with $1 \times$ DPBS and stained with $0.5 \%$ crystal violet for 15 mins at room temperature. The cells were washed free of any stain and photographed with a digital camera.

\section{Wound healing assay}

A549 cells were seeded (approximately $1 \times 10^{6}$ cells) in wells of a 6-well plate until monolayers formed. The cell layers were scratched with a sterile micropipette tip (Sigma-Aldrich), washed three times with $1 \times$ DPBS, and incubated in a fresh serum-free RPMI-1640 medium with or without K-AuNCs for upto $24 \mathrm{hrs}$. The number of migrating cells was observed under an inverted microscope and photographed at 0 and $24 \mathrm{hrs}$ after scratching. 


\section{Statistical analysis}

All experiments were performed in replicates of three. The results are presented as means \pm standard deviations (SDs). The statistical significance was evaluated using Student's $t$ test. $P$-values $<0.05$ were considered significant.

\section{Result and discussion}

\section{Synthesis of K-AuNCs}

$\mathrm{K}$-AuNCs were synthesized by allowing kaempferol to react with $\mathrm{HAuCl}_{4}$ and $\mathrm{BSA}$. After addition of $\mathrm{NaOH}$, the color change indicated the formation of K-AuNCs. BSA was used to control the size of the clusters in the quantum-sized material during the growth and nucleation processes with a high fluorescence quantum yield of $11 \%{ }^{16,17}$ Kaempferol is a potent-reducing agent and $\mathrm{NaOH}$ was also used as reducing agent for the formation

A

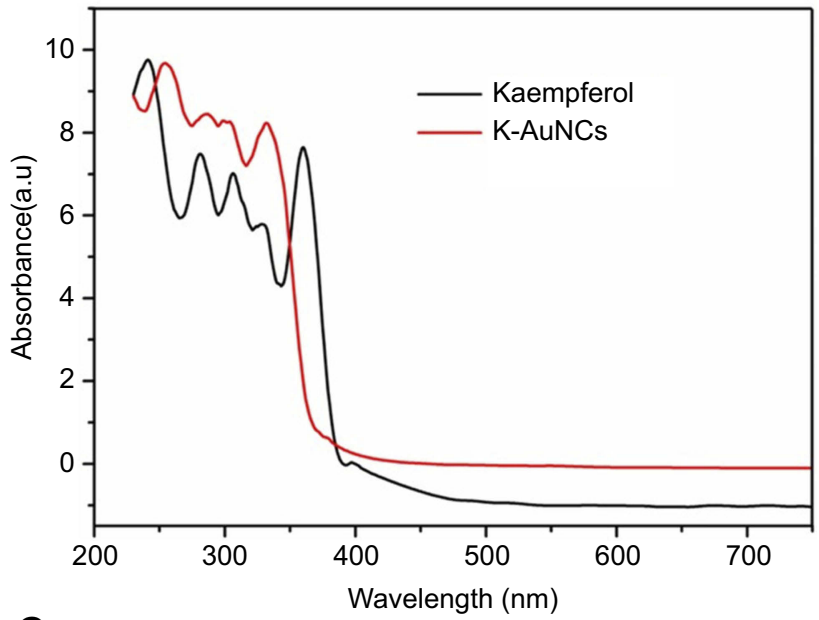

C

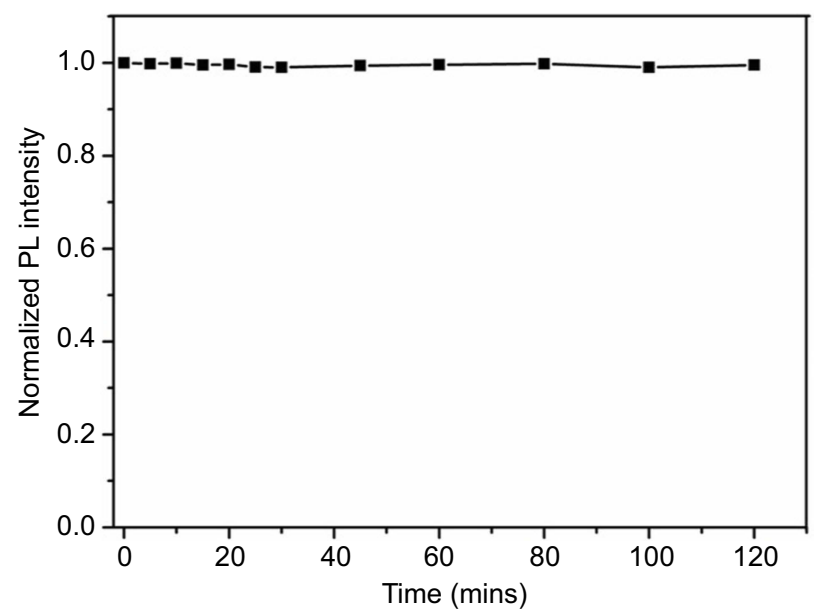

of K-AuNCs. ${ }^{18}$ Vigorous stirring was used to reduce most of the $\mathrm{AU}$ atoms from $\mathrm{Au}^{3+}$ to $\mathrm{Au}^{1+}$ in the presence of kaempferol and BSA. $\mathrm{NaOH}$ completed the reduction of $\mathrm{Au}^{1+}$ to $\mathrm{Au}^{\circ}$ with the use of heat and stirring for the appropriate time. $^{19}$

\section{Optical property of K-AuNCs}

The UV-Vis absorbance and PL spectra were analyzed for the synthesized K-AuNCs. The UV spectra also included pure kaempferol (Figure 1A). Pure kaempferol displayed a small peak at $335 \mathrm{~nm}$ and another large peak at $366 \mathrm{~nm}$. The same peaks were evident after formation of $\mathrm{K}$-AuNCs with a slight shift toward the blue region of the spectrum (approximately at 300 $\mathrm{nm}$ and $350 \mathrm{~nm}$ ), confirming the presence of kaempferol in the K-AuNCs. A small peak at $280 \mathrm{~nm}$

B

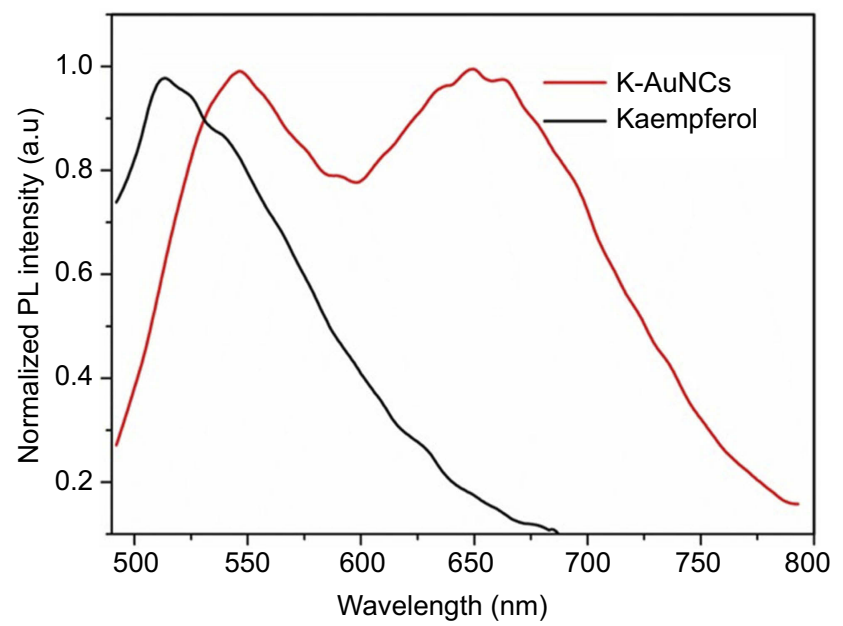

D

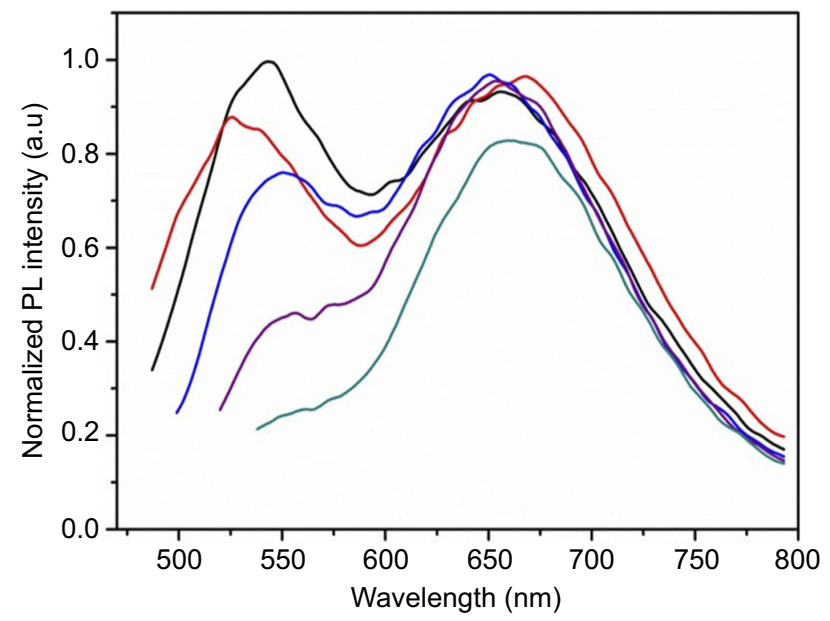

Figure I Optical characterizations of K-AuNCs: (A) UV-Vis spectrum of kaempferol and K-AuNCs. (B) PL emission spectra of kaempferol and K-AuNCs. Fluorescence stability of K-AuNCs from 0-120 min and (D) image is emission peak of K-AuNCs corresponding to the different excitation wavelength. 
indicates the combining of BSA with $\mathrm{Au}$ ions. ${ }^{20}$ No large absorbance peak was observed in the UV-Vis spectra due to the small size of the K-AuNCs. The light and generate of SPR were interacted by the electron conduction by the K-AuNCs. Particles in the quantum range have distinguishable properties because the space between the energy levels is inversely proportional to the size of the particles. ${ }^{21}$ The PL intensity of pure kaempferol and the synthesized K-AuNCs were analyzed (Figure 1B). The peak at $\sim 510 \mathrm{~nm}$ represents the emission spectra of pure kaempferol. After the conjugation of K-AuNCs, the kaempferol peak shifted by approximately $30 \mathrm{~nm}$ to the red shift at $550 \mathrm{~nm}$. The shift confirmed the conjugation of kaempferol to the KAuNCs. The maximum excitation peak was observed at $450 \mathrm{~nm}$ and the emission peak was approximately 650 $\mathrm{nm}$ for the K-AuNCs. The inset images in Figure 1B, (a) represents the K-AuNCs in visible light and (b) shows the K-AuNCs under the UV light at $365 \mathrm{~nm}$.

Fluorescence stability of the K-AuNCs was analyzed using the PL spectra (Figure 1C). The fluorescence property of the K-AuNCs was very stable for up to $100 \mathrm{mins}$ at room temperature (Figure 1C) and also the material is stable up to six months stored was in $4^{\circ} \mathrm{C}$ (Figure S1) and the PL intensity remained the same. Excitation was explored using wavelengths ranging from $420 \mathrm{~nm}$ to $500 \mathrm{~nm}$ (Figure 1D). The fluorescent intensity remained in the same shift at $650 \mathrm{~nm}$ and there was no change in the intensity corresponding to the different excitation wavelengths.

A

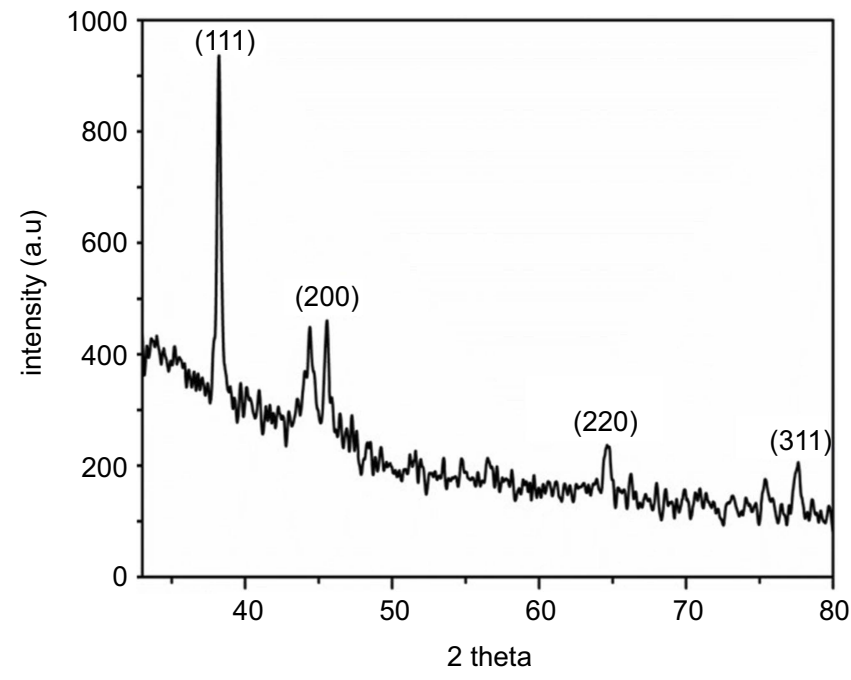

\section{X-ray diffraction (XRD) and FTIR spectroscopy}

The XRD observations are presented in Figure 2A. The crystallinity and diffracted angles of the K-AuNCs were evaluated from $36^{\circ}$ to $80^{\circ}$. The diffraction peaks at $45^{\circ}$ and $65^{\circ}$ corresponded to the (200) and (220) lattice planes. The diffraction peak at $38^{\circ}$ and $78^{\circ}$ was indexed to the (111) and (311) reflection of the face-centered cube (fcc) structure of gold. ${ }^{10,22}$ FTIR analyses were carried out to identify the functional groups present in the products and the chemical interaction between kaempferol and K-AuNCs in Figure 2B. (i) Pure kaempferol and (ii) is K-AuNCs. The broad band at $3309 \mathrm{~cm}^{-1}$ corresponding to the phenolic hydroxyl $(\mathrm{OH})$. Another band at approximately $1660 \mathrm{~cm}^{-1}$ represented the stretching vibrations of $\mathrm{C}=\mathrm{O}$ groups in the $\mathrm{C}$ ring of the flavonoid structure. ${ }^{23,24}$ Several stretching bands at approximately $1048,1607,1503$, and $1448 \mathrm{~cm}^{-1}$ corresponded to the benzopyran group and aromatic skeleton. The $\mathrm{OH}$ stretching band shifted slightly to $3396 \mathrm{~cm}^{-1}$, and the same benzopyran group remained in $\mathrm{K}-\mathrm{AuNCs}$, confirming the presence of kaempferol in the K-AuNCs. A similar band was observed for other bioflavonoids conjugated with AuNPs. ${ }^{24}$

\section{Morphological analysis of K-AuNCs}

The morphological analyses of the synthesized K-AuNCs were performed using HR-TEM and bio-atomic force microscopy (AFM) (Figure 3). The synthesized NCs were uniform in size and were well dispersed. HR-TEM

B

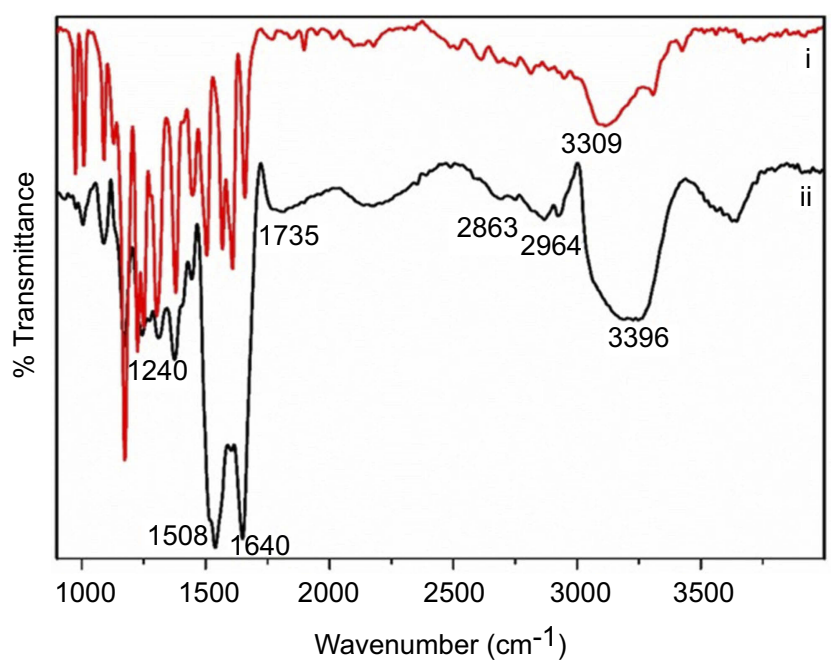

Figure 2 (A) X-ray diffraction spectrum of K-AuNCs and (B) FTIR characterization of kaempferol and K-AuNCs. (i) Pure kaempferol and (ii) is K-AuNCs. 
A

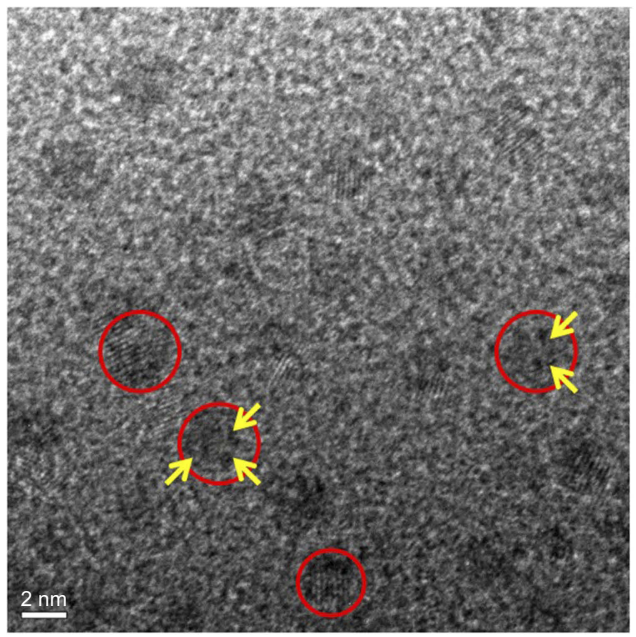

B

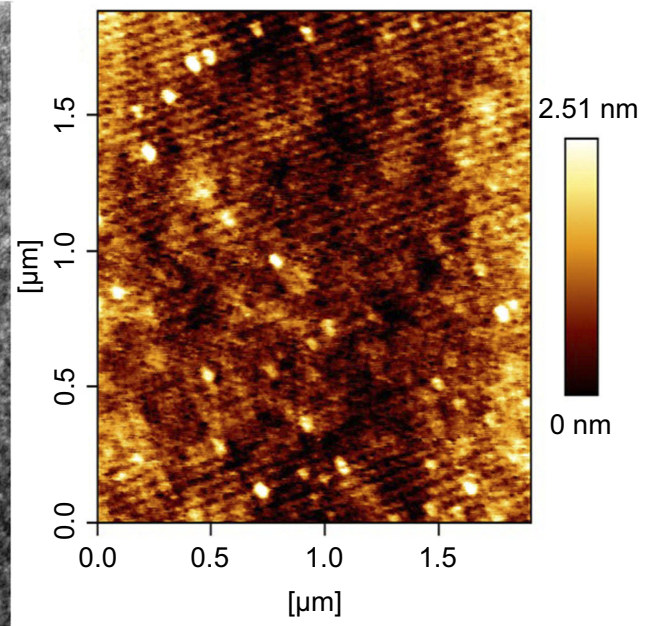

Figure 3 Morphological characterization of K-AuNCs. (A) HR-TEM analysis of K-AuNCs. (B) Bio-AFM height images of K-AuNCs. Notes: Red circles indicate the formation of K-AuNCs and the yellow arrows indicate the Au crystals were organized well.

images clearly showed that the K-AuNCs were $1-3 \mathrm{~nm}$ in size; the minimum size of the K-AuNCs is $1 \mathrm{~nm}$ and maximum size is $3 \mathrm{~nm}$. The small atoms combined together to form the NCs (Figure 3A) and the red circle in the figure indicates the clusters and the yellow arrow denotes the organized NCs. ${ }^{15}$ HR-TEM clearly revealed the crystallinity of the K-AuNCs in the fcc structure of Au. The lattice fringe spacing of K-AuNCs could represent the (111) reflection of the fcc structure of Au. The average height of the synthesized K-AuNCs analyzed by Bio-AFM was approximately $2.5 \mathrm{~nm}$, and particles were well dispersed and uniform in size (Figure 3B).

\section{Cytotoxicity of K-AuNCs}

The MTT assay was performed to determine the cytotoxicity of K-AuNCs to A549 cells and HK-2 normal human kidney cells (Figure 4), based on a previous report that kaempferol is cytotoxic to lung cancer cells. ${ }^{25} \mathrm{~K}$-AuNCs concentrations of 12.5 and $25 \mu \mathrm{g} / \mathrm{mL}$ were treated with HK-2 normal human kidney cells and A549 lung cancer cells. The percentage of

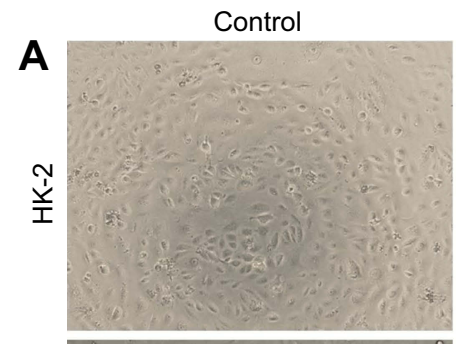

B

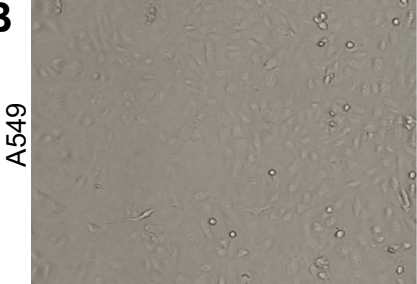

$12.5 \mu \mathrm{g} / \mathrm{mL}$
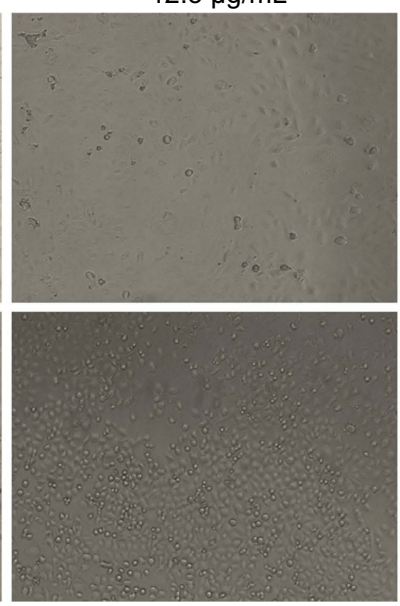

$25 \mu \mathrm{g} / \mathrm{mL}$
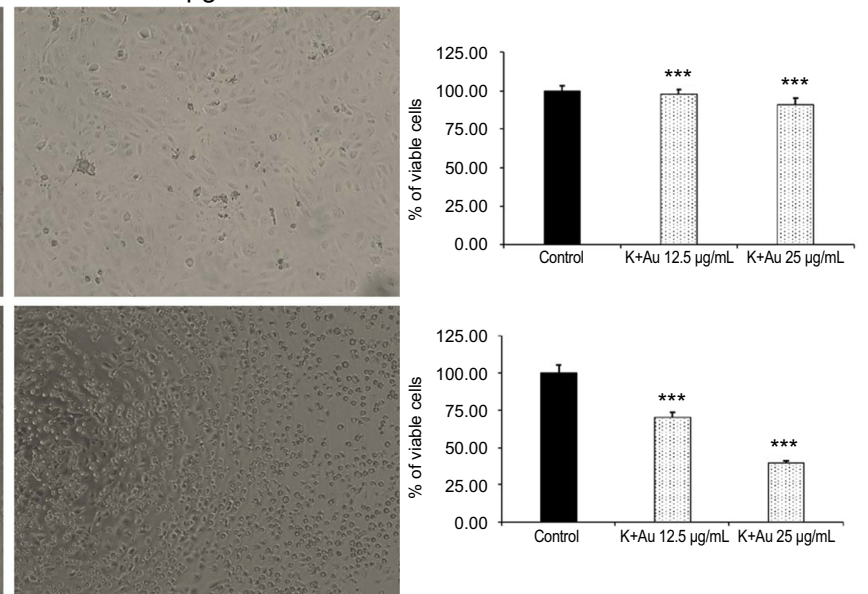

Figure 4 MTT assay to evaluate the cytotoxicity of K-AuNCs on HK-2 human normal kidney cells and A549 lung cancer cell line. Two different concentrations of the formulations namely, $12.5 \mu \mathrm{g} / \mathrm{mL}$ and $25 \mu \mathrm{g} / \mathrm{mL}$ were treated to the normal and lung cancer cells. Cells were then incubated for up to 24 hrs. MTT reagent was added to each well and further incubated in dark for $4 \mathrm{hrs}$. Following this, the OD was recorded. The percentage of viable cells in each group was calculated and plotted as graph. (A) HK-2 human kidney cell line showed very less cytotoxicity (B) A549 human lung cancer cell line on the other hand showed significant increase in cytotoxicity with increasing concentrations of K-AuNCs. Each value represents the mean \pm SE $(n=5) * p<0.05$. Scale bar $=200 \mu m$.

Note: ${ }^{* * *} p<0.001$. 
viable cells after $24 \mathrm{hrs}$ of treatment was calculated. Consistent with the previous report, the MTT assay also revealed the cytotoxicity of the K-AuNCs to the A549 lung cancer cells. However, HK-2 cells were not affected at these concentrations. Therefore, K-AuNCs were effective in selectively inducing cytotoxicity to the cancer cells while leaving the normal cells unharmed (Figure 4A and B). The cytotoxicity was evident at a very low concentration $(12.5 \mu \mathrm{g} / \mathrm{mL})$ in the lung cancer cells. The same concentration did not affect HK-2 cells. Moreover, Annexin staining were performed to show the apoptotic cells in the control vs K-AuNCs treated A549 lung cancer cells (Figure S2). Figure S2A shows Annixin V-FITC/ PI double staining of apoptosis in A549 control or K-AuNCs $(12.5 \mu \mathrm{g} / \mathrm{mL})$ treated cells. Table represents the percentage of cells live and in different stages of apoptosis. After the treating with K-AuNCs, $21.8 \%$ of the cells were present in the apoptosis and $70.8 \%$ of the cells were present in the late apoptosis. Cells were treated or untreated with appropriate concentrations of the NCs for $24 \mathrm{hrs}$ and followed by standard Annexin staining protocol.

\section{K-AuNCs induced nuclear damage}

Condensed and fragmented nuclei are characteristics of apoptosis. Nuclear damage has been reported upon treatment of cancer cells with K-AuNCs in the confocal imaging of DAPI-stained samples. ${ }^{26,27}$ Nuclear damage upon drug treatment in cancer cells may be caused by several different molecular mechanisms. Kaempferol is a potent anti-cancer drug that causes DNA damage in several types of cancer cells. $^{27-31}$ A549 lung cancer cells were treated with KAuNCs for $24 \mathrm{hrs}$. Cell nuclei were stained with DAPI. The nuclear morphology of the untreated cells vs cells treated with K-AuNCs was evaluated using confocal microscopy.
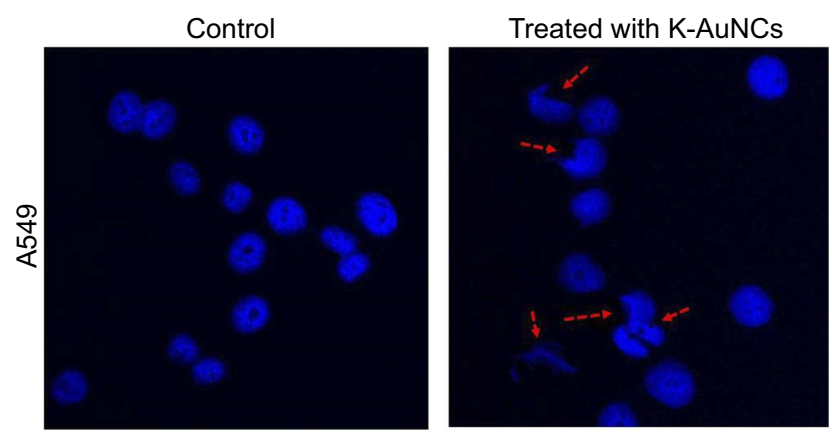

Figure 5 Nuclear damage indicating cell death was evaluated in A549 human lung cancer cell line treated with K-AuNCs. Accordingly, the cells were treated with the desired concentration of K-AuNCs and incubated for $24 \mathrm{hrs}$. Cells were then fixed and stained with DAPI to visualize the nucleus. A549 cells showed increased nuclear damage upon treatment with K-AuNCs indicating apoptosis in the treated cells. Scale bar $=50 \mu \mathrm{m}$.
The morphology of the untreated cells remained intact and regular, while damaged nucleus was seen in lung cancer cells treated with $\mathrm{K}-\mathrm{AuNCs}$, indicating nuclear breakage, indicating apoptosis in these cells (Figure 5).

\section{K-AuNCs controlled proliferation of lung cancer cells}

Uncontrolled cell proliferation is an important hallmark of cancer. $^{32}$ To evaluate the regulation of lung cancer cell proliferation by K-AuNCs, a clonogenic assay was performed. A549 human lung cancer cells were untreated or treated with

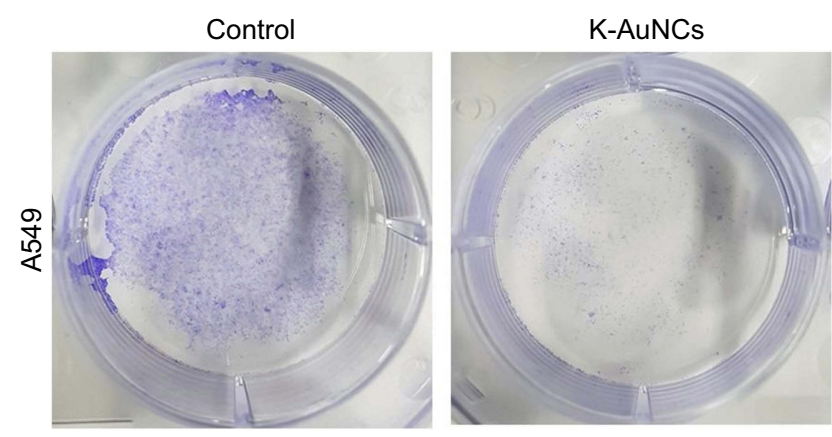

Figure 6 K-AuNCs inhibited the proliferation of A549 human lung cancer cells. Cells were assessed for proliferation with colony formation assay. Accordingly, cells were seeded on a $60-\mathrm{mm}$ dish. Cells were then treated with or without K-AuNCs formulation and incubated further for 2 weeks. Cells were then stained with Crystal Violet and the number of colonies formed in control and K-AuNCs treated groups were observed. K-AuNCs treatment drastically reduced the cell proliferation and number of colonies formed by A549 human lung cancer cell line.
$\mathrm{Oh}$

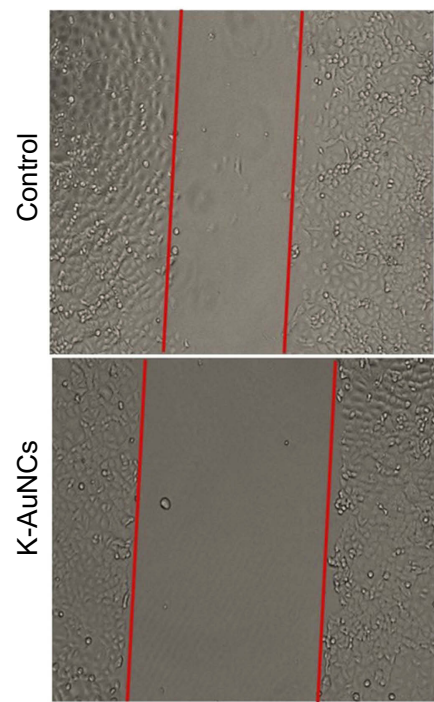

$24 \mathrm{~h}$

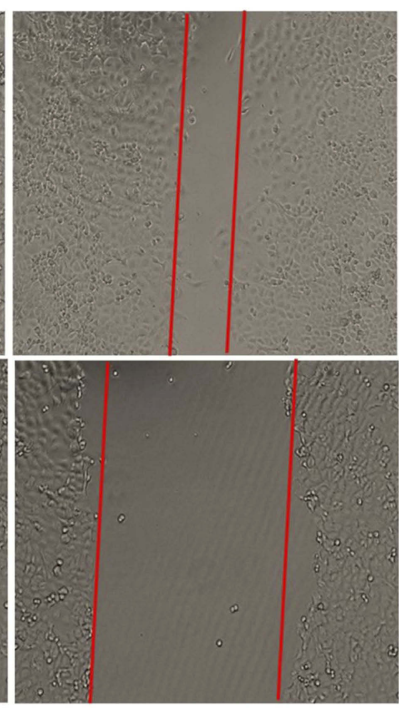

Figure $7 \mathrm{~K}-\mathrm{AuNCs}$ inhibited the migration of A549 human lung cancer cells. Cells were assessed for migration with wound healing assay. Accordingly, cells were seeded and cultured until confluence was reached. A scratch was made and cells were treated with or without K-AuNCs formulation.The area of the wound was evaluated at $\mathrm{Oh}$ and $24 \mathrm{~h}$ in control and treated cells.

Note: $* p<0.05$. 
$\mathrm{Oh}$
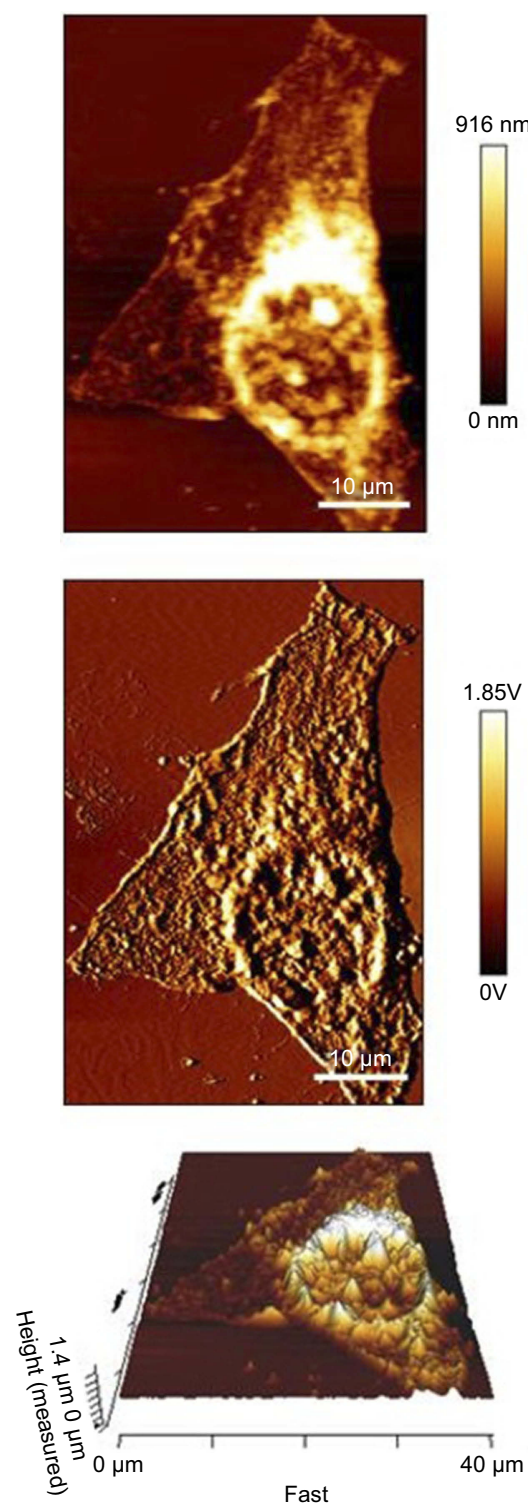

$12 \mathrm{~h}$
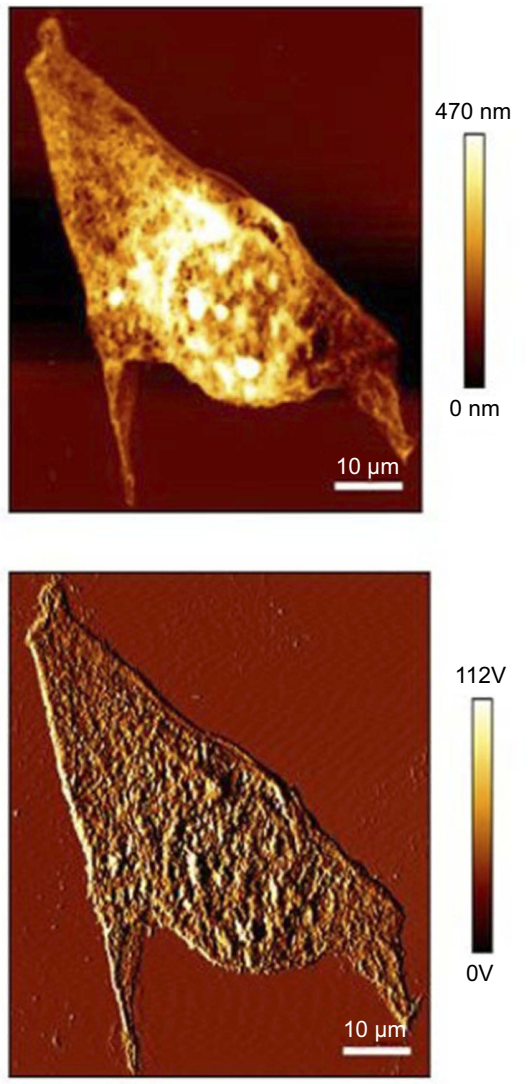

$24 \mathrm{~h}$
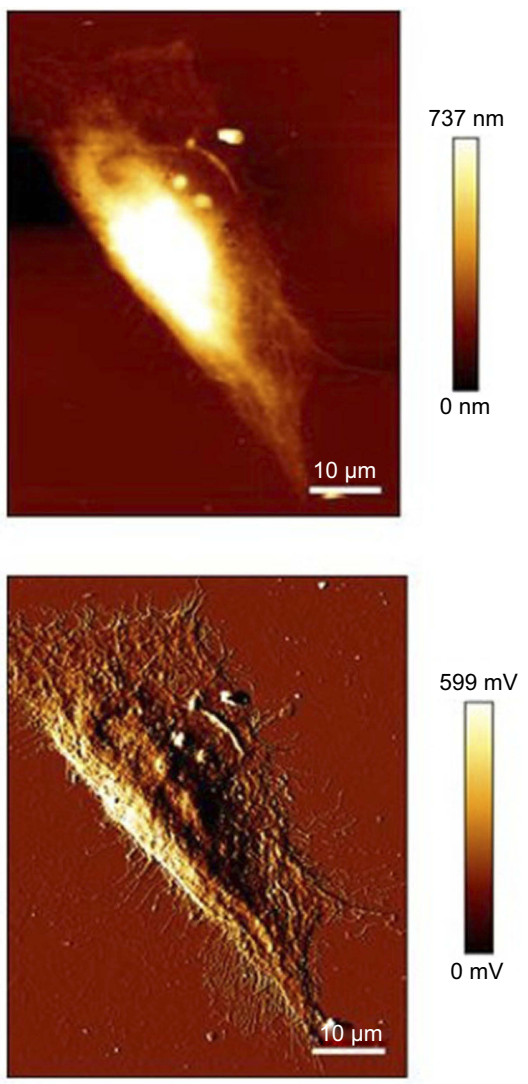
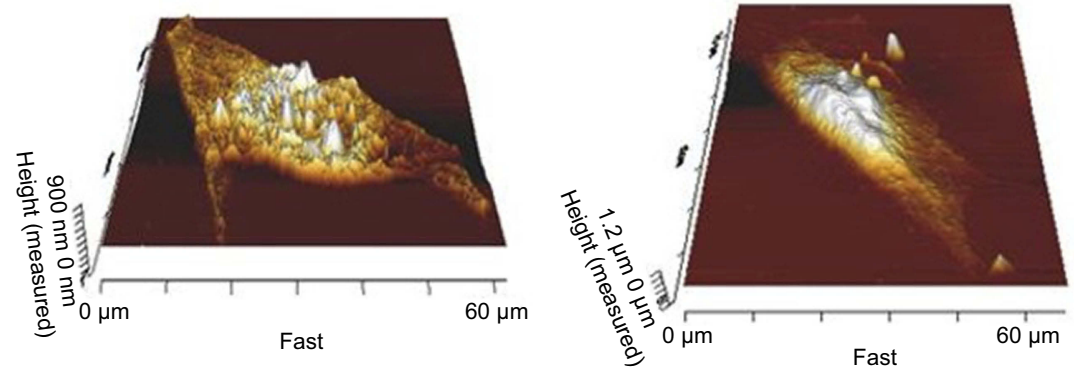

Figure 8 Bio-AFM height, error and 3D images of A549 calls treated with K-AuNCs in different intervals of time from 0, I2, and 24 hrs.

$\mathrm{K}$-AuNCs for $24 \mathrm{hrs}$ and the number of colonies formed was evaluated after two weeks. Control untreated cells formed many colonies, while significantly fewer colonies were evident in the K-AuNCs-treated samples. These results indicated that K-AuNCs can effectively inhibit the proliferation of human lung cancer cells (Figure 6).

\section{K-AuNCs control migration of lung cancer cells}

Kaempferol is an active flavonoid with potent anti-cancer and anti-migratory properties in different types of cancer cells. ${ }^{33,34}$ We evaluated if $\mathrm{K}-\mathrm{AuNC}$ conferred similar anti-migratory effects on cancer cells by evaluating the regulation of the A549 lung cancer cell migration at 0 and $24 \mathrm{hrs}$ following treatment with $12.5 \mu \mathrm{g} / \mathrm{mL}$ $\mathrm{K}$-AuNCs using the scratch assay. The images were captured to evaluate the recovery of the scratch area. The number of migrating cells at 0 and $24 \mathrm{hrs}$ in the treatment groups were less compared to the control untreated cells. The K-AuNCs drastically inhibited the migration of A549 cells. The wound was not significantly sealed for up to $24 \mathrm{hrs}$. However, the control untreated cells migrated and the wound area was reduced in size at 24 
hrs (Figure 7). The percentage of wound area at $0 \mathrm{hr}$ to 24 hrs compared with the control and treated cells was validated. These results indicated the K-AuNCs-mediated inhibition of A549 cell migration.

\section{Morphology of A549 cells treated with K-AuNCs}

To better understand how the K-AuNCs reacted with A549 cells with time $(0,12$, and $24 \mathrm{hrs})$ and to monitor the morphology change and damage of cell nuclei, cells were monitored using Bio-AFM (Figure 8). The deflection images revealed that nuclear damage and surface morphology change began in the K-AuNCs-treated cells within 12 hrs. After 24 hrs, cell damage was extensive and uneven, and was followed by cell death. The results supported the anti-tumor activity of K-AuNCs for A549 cells.

\section{Conclusions}

K-AuNCs were successfully synthesized and its anticancer properties were studied in A549 lung cancer cells. The synthesized K-AuNCs exhibited strong red fluorescence and were stable for up to 100 mins. The size of the $\mathrm{K}$-AuNCs is $1-3 \mathrm{~nm}$ and the nanoclusters are perfectly dispersed. Kaempferol could be efficiently conjugated to the AuNCs, and the conjugate was selectively toxic to the lung cancer and was not toxic to HK-2 normal human kidney cells. Damage to the cancer cells included the outer surface and nucleus, and the damage was evident at 12.5 and $25 \mu \mathrm{g} / \mathrm{mL}$ after 12 and $24 \mathrm{hrs}$. The clonogenic assay confirmed that $\mathrm{K}-\mathrm{AuNCs}$ controlled the proliferation of A549 cells. A549 cell migration was explored using untreated cells and cells treated with $12.5 \mu \mathrm{g} / \mathrm{mL}$ K-AuNCs for 0-24 hrs. K-AuNCs impeded migration. The results support the anticancer efficacy of the natural flavonoid kaempferol when conjugated to AuNCs. The strong fluorescence of the conjugate allows bioimaging applications.

\section{Acknowledgments}

This research was supported by the National Research Foundation of Korea (NRF) grant funded by the Korea Government (No.2017R1A2B4004700). This work was supported by the Ministry of Trade, Industry, and Energy (Grant No.20000580).

\section{Disclosure}

The authors report no conflicts of interest in this work.

\section{References}

1. Yu R, Zou Y, Liu B, Guo Y, Wang X, Han M. Surface modification of $\mathrm{pH}$-sensitive honokiol nanoparticles based on dopamine coating for targeted therapy of breast cancer. Colloids Surf B. 2019;177:1-10. doi:10.1016/j.colsurfb.2019.01.047

2. Danhier F, Feron O, Préat V. To exploit the tumor microenvironment: passive and active tumor targeting of nanocarriers for anti-cancer drug delivery. J Control Release. 2010;148:135-146. doi:10.1016/j. jconrel.2010.08.027

3. Janganati V, Ponder J, Thakkar S, Jordan CT, Crooks PA. Succinamide derivatives of melampomagnolide B and their anticancer activities. Bioorganic Med Chem. 2017;25:3694-3705. doi:10.1016/j.bmc.2017.05.008

4. Du L, Qin H, Ma T, Zhang T, Xing D. In vivo imaging-guided photothermal/photoacoustic synergistic therapy with bioorthogonal metabolic glycoengineering-activated tumor targeting nanoparticles. ACS Nano. 2017;11:8930-8943. doi:10.1021/acsnano.7b03226

5. Wen L, Yang S, Zhong J, Zhou Q, Xing D. Thermoacoustic imaging and therapy guidance based on ultra-short pulsed microwave pumped thermoelastic effect induced with superparamagnetic iron oxide nanoparticles. Theranostics. 2017;7:1976-1989. doi:10.7150/thno.17846

6. Wei Y, Zhou F, Zhang D, Chen Q, Xing D. A graphene oxide based smart drug delivery system for tumor mitochondria-targeting photodynamic therapy. Nanoscale. 2016;8:3530-3538. doi:10.1039/ c5nr07785k

7. Qin H, Zhou T, Yang S, Xing D. Fluorescence quenching nanoprobes dedicated to in vivo photoacoustic imaging and high-efficient tumor therapy in deep-seated tissue. Small. 2015;11:26752686. doi:10.1002/smll.201403395

8. Somerset SM, Johannot L. Dietary flavonoid sources in Australian adults. Nutr Cancer. 2008;60:442-449. doi:10.1080/ 01635580802143836

9. Chen AY, Chen YC. A review of the dietary flavonoid, kaempferol on human health and cancer chemoprevention. Food Chem. 2013;138:2099-2107. doi:10.1016/j.foodchem.2012.11.139

10. Raghavan BS, Kondath S, Anantanarayanan R, Rajaram R. Kaempferol mediated synthesis of gold nanoparticles and their cytotoxic effects on MCF-7 cancer cell line. Process Biochem. 2015;50:1966-1976. doi:10.1016/j.procbio.2015.08.003

11. Thangavel P, Viswanath B, Kim S. Synthesis and characterization of kaempferol-based ruthenium (II) complex: a facile approach for superior anticancer application. Mater Sci Eng C. 2018;89:87-94. doi:10.1016/j.msec.2018.03.020

12. Li Z, Liu Y, Huang X, et al. One-step preparation of gold nanovectors using folate modified polyethylenimine and their use in target-specific gene transfection. Colloids Surf B. 2019;177:306-312. doi:10.1016/j.colsurfb.2019.02.011

13. Yang Y, Nan J, Hou J, et al. Cytotoxicity of gold nanoclusters in human liver cancer cells. Int $J$ Nanomed. 2014;9:5441-5448. doi:10.2147/IJN.S69013

14. Zhang W, Lin D, Wang H, et al. Supramolecular self-assembly bioinspired synthesis of luminescent gold nanocluster-embedded peptide nanofibers for temperature sensing and cellular imaging. Bioconjugate Chem. 2017;28:2224-2229. doi:10.1021/acs. bioconjchem. 7b00312

15. Govindaraju S, Rengaraj A, Arivazhagan R, Huh YS, Yun K. Curcumin-conjugated gold clusters for bioimaging and anticancer applications. Bioconjugate Chem. 2018;29:363-370. doi:10.1021/ acs.bioconjchem.7b00683

16. Wang Y, Chen J, Irudayaraj J. Nuclear targeting dynamics of gold nanoclusters for enhanced therapy of HER2+ breast cancer. ACS Nano. 2011;5:9718-9725. doi:10.1021/nn2032177

17. Xie J, Zheng Y, Ying JY. Protein-directed synthesis of highly fluorescent gold nanoclusters. J Am Chem Soc. 2009;131:888-889. doi:10.1021/ja806804u 
18. Halder A, Das S, Bera T, Mukherjee A. Rapid synthesis for monodispersed gold nanoparticles in kaempferol and anti-leishmanial efficacy against wild and drug resistant strains. RSC Adv. 2017;7:1415914167. doi:10.1039/C6RA28632A

19. Negishi Y, Nobusada K, Tsukuda T. Glutathione-protected gold clusters revisited: bridging the gap between gold (I) - thiolate complexes and thiolate-protected gold nanocrystals. $J$ Am Chem Soc. 2005;127:5261-5270. doi:10.1021/ja042218h

20. Ding C, Tian Y. Gold nanocluster-based fluorescence biosensor for targeted imaging in cancer cells and ratiometric determination of intracellular pH. Biosens Bioelectron. 2015;65:183-190. doi:10.1016/j.bios.2014.10.034

21. Qu X, Li Y, Li L, Wang Y, Liang J, Liang J. Fluorescent gold nanoclusters: synthesis and recent biological application. J Nanomater. 2015;2015:23. doi:10.1155/2015/784097

22. Krishnamurthy S, Esterle A, Sharma NC, Sahi SV. Yucca-derived synthesis of gold nanomaterial and their catalytic potential. Nanoscale Res Lett. 2014;9:627. doi:10.1186/1556-276X-9-627

23. Bellamy LJ, editor. Amides proteins polypeptides. In: In the InfraRed Spectra of Complex Molecules. Dordrecht: Springer; 1975:231262. doi:10.1007/978-94-011-6017-9 12

24. Levchenko LA, Golovanova SA, Lariontseva NV, et al. Synthesis and study of gold nanoparticles stabilized by bioflavonoids. Russ Chem B. 2011;60:426. doi:10.1007/s11172-011-0067-1

25. Nguyen TTT, Tran E, Ong CK, et al. Kaempferol-induced growth inhibition and apoptosis in A549 lung cancer cells is mediated by activation of MEK-MAPK. J Cell Physiol. 2003;197:110-121. doi:10.1002/jcp. 10340

26. Stepanić V, Kujundžić RN, Troselj KG. Epigenome, cancer prevention and flavonoids and curcumin. In: Payne CJ, editor. Epigenetics and Epigenomics. InTech; 2014:173-210.
27. Bestwick CS, Milne L, Pirie L, Duthie SJ. The effect of short-term kaempferol exposure on reactive oxygen levels and integrity of human (HL-60) leukaemic cells. Biochim Biophys Acta. 2005;1740:340-349. doi:10.1016/j.bbadis.2004.10.005

28. Wu LY, Lu HF, Chou YC, et al. Kaempferol induces DNA damage and inhibits DNA repair associated protein expressions in human promyelocytic leukemia HL-60 cells. Am $J$ Chin Med. 2015;43:365-382. doi:10.1142/S0192415X1550024X

29. Tiwari P. Role of flavonoids in DNA damage and carcinogenesis prevention. J Carcinog Mutagen. 2017;8:2157-2518. doi:10.4172/ 2157-2518.1000297

30. Song W, Dang Q, Xu D, et al. Kaempferol induces cell cycle arrest and apoptosis in renal cell carcinoma through EGFR/p38 signaling. Oncol Rep. 2014;31:1350-1356. doi:10.3892/or.2014.2965

31. Rajendran P, Rengarajan T, Nandakumar N, Palaniswami R, Nishigaki Y, Nishigaki I. Kaempferol a potential cytostatic and cure for inflammatory disorders. Eur J Med Chem. 2014;86:103-112. doi:10.1016/j.ejmech.2014.08.011

32. Hanahan D, Weinberg RA. Hallmarks of cancer: the next generation. Cell. 2011;144:646-674. doi:10.1016/j.cell.2011.02.013

33. Li S, Yan T, Deng R, et al. Low dose of kaempferol suppresses the migration and invasion of triple-negative breast cancer cells by downregulating the activities of rhoa and rac1. Onco Targets Ther. 2017;10:4809. doi:10.2147/OTT.S140886

34. Hung TW, Chen PN, Wu HC, et al. Kaempferol inhibits the invasion and migration of renal cancer cells through the downregulation of AKT and FAK pathways. Int J Med Sci. 2017;14:984. doi:10.7150/ ijms. 20336 


\section{Supplementary materials}

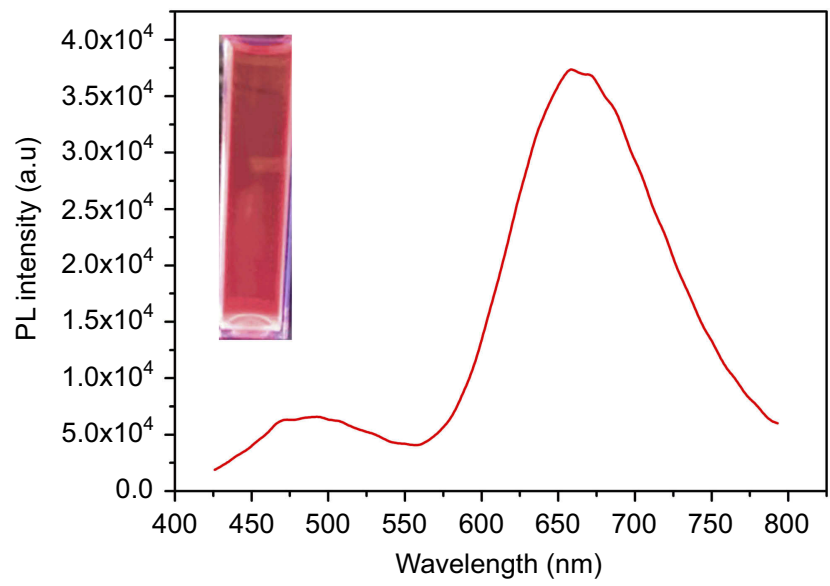

Figure SI Fluorescence stability of the K-AuNCs was checked with six months before synthesized sample, and it was stored in $4^{\circ} \mathrm{C}$. The synthesized material gives good fluorescence intensity. The inset image indicated that red color fluorescence is present under the UV light for the same sample.

A

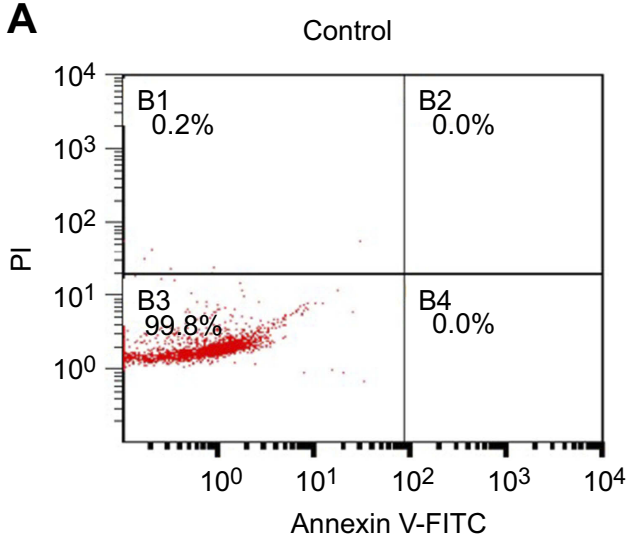

B

\begin{tabular}{|l|c|c|c|c|}
\hline Treatment & $\begin{array}{c}\text { Live cell } \\
(\%)\end{array}$ & $\begin{array}{c}\text { Apoptosis } \\
\text { cell (\%) }\end{array}$ & Late apoptosis cell (\%) & $\begin{array}{c}\text { Necrosis } \\
\text { cell (\%) }\end{array}$ \\
\hline Control & 99.8 & 0.0 & 0.0 & 0.2 \\
\hline K-AuNCs & 7.4 & 21.8 & 70.8 & 0.0 \\
\hline
\end{tabular}

Figure S2 (A) Annixin V-FITC/PI double staining of apoptosis in A549 control or K-AuNCs ( $12.5 \mu g / m L)$ treated cells. (B) Table represents the percentage of cells live and in different stages of apoptosis.

International Journal of Nanomedicine

\section{Dovepress}

\section{Publish your work in this journal}

The International Journal of Nanomedicine is an international, peerreviewed journal focusing on the application of nanotechnology in diagnostics, therapeutics, and drug delivery systems throughout the biomedical field. This journal is indexed on PubMed Central, MedLine, CAS, SciSearch ${ }^{\mathbb{B}}$, Current Contents $\mathrm{s}^{\mathbb{B}} /$ Clinical Medicine,

Journal Citation Reports/Science Edition, EMBase, Scopus and the Elsevier Bibliographic databases. The manuscript management system is completely online and includes a very quick and fair peer-review system, which is all easy to use. Visit http://www.dovepress.com/ testimonials.php to read real quotes from published authors. 